\title{
A Phase II Study of Antineoplastons A10 and AS2-1 Injections in Adult Patients With Recurrent Anaplastic Astrocytoma-Final report (Protocol BT-15)
}

\author{
Stanislaw R. Burzynski ${ }^{1}$, Tomasz J. Janicki ${ }^{1} \&$ Gregory S. Burzynski ${ }^{1}$ \\ ${ }^{1}$ Burzynski Clinic, 9432 Katy Freeway, Houston, Texas 77055, USA \\ Correspondence: Stanislaw R. Burzynski, Burzynski Clinic, 9432 Katy Freeway, Houston, Texas 77055, USA. \\ Tel: 1-713-335-5697. E-mail: srb@burzynskiclinic.com
}

Received: April 9, 2015

Accepted: May 7, 2015 Online Published: June 17, 2015

doi:10.5539/cco.v4n2p13

URL: http://dx.doi.org/10.5539/cco.v4n2p13

\begin{abstract}
The recommended treatment for recurrent anaplastic astrocytoma (AA) consists of chemotherapy with temozolomide. There is an urgent need for new therapies for such patients. This phase II study evaluates efficacy and safety of a different form of cancer therapy that consists of antineoplastons A10 and AS2-1 (ANP). 27 patients, ages 19 to 57 years (median, 41) with recurrent AA were treated. All patients previously underwent surgery, all received radiation therapy, and 16 underwent chemotherapy. The patients received treatment with ANP administered daily every four hours (median dose of A10 $6.0 \mathrm{~g} / \mathrm{kg} / \mathrm{d}$ and AS2-1 $0.25 \mathrm{~g} / \mathrm{kg} / \mathrm{d}$ ). All patients were included in efficacy and safety analysis. The median duration of ANP was 23.1 weeks. A complete response was documented in $7 \%$, partial response in $11 \%$, and stable disease in $22 \%$ of patients. Progression-free survival and overall survival at 12 months was $26 \%$ and $41 \%$ respectively. The only serious toxicity was Grade 3 reversible hypokalemia in $7 \%$ of patients. There was no chronic toxicity and there was a high quality of life. ANP shows efficacy with very good toxicity profile in this cohort of patients with recurrent AA.
\end{abstract}

Keywords: anaplastic astrocytoma, Antineoplastons A10 and AS2-1, brain tumor, phase II clinical trial, recurrent glioma, temozolomide

\section{Introduction}

Anaplastic astrocytoma (AA) account for approximately 5,600 people in the United States this year (Ostrom et al., 2014). Classified as a World Health Organization grade 3 neuroepithelial tumor (Louis et al., 2007), AA is also known as malignant astrocytoma or high-grade astrocytoma. Initial standard therapy for brain tumors includes the surgical resection whenever possible within the constraints of preservation of neurologic function. After surgery, AA patients routinely undergo radiation therapy and/or chemotherapy. Given the ability of these tumors to relapse, surgery alone is not considered a reasonable means of treatment. Historically, nitrosourea therapy provided the first successful treatment for AA, but within a decade temozolomide (TMZ) became the most frequently used therapeutic agent due to better toxicity profile and comparable efficacy (Yung et al., 1999; Dinnes, Cave, Huang, \& Milne, 2002). TMZ gave a 20\% response rate in patients who had previously received nitrosourea-based therapy, prompting the Food and Drug Administration (FDA) to grant accelerated approval for the use of TMZ in the treatment of recurrent AA (Mehta, Vogelbaum, Chang, \& Patel, 2011). Clinical observations confirmed that the remissions from treatment with TMZ were temporary, resulting in progression-free survival (PFS) of $24 \%$ at 12 months (Yung et al., 1999). Ultimately almost all patients would relapse and die from progressive disease. It was soon realized that it is necessary to test new therapeutic regimens for patients who relapse after nitrosourea and TMZ. From January 1994 until summer of 2012, there were nine phase II clinical studies conducted for patients with recurrent AA with the results reported in peer-reviewed journals in English. There was also a single additional report in Japanese (Nishikawa et al., 2006). There were only two phase II trials exclusively designed for AA patients and both of them failed to prove efficacy (Chamberlain, Tsao-Wei, \& Groshen, 2006; Chamberlain, Tsao-Wei, Blumenthal, \& Glantz, 2008). Seven studies accrued only from 1 to 15 AA patients (Robins et al., 2002; Spence, Peterson, Scharnhorst, Silbergeld, \& Rostomily, 2004; Kirby et al., 2005; Neyns et al., 2008; Franceschi et al., 2007; de Groot et al., 2011; Bogdahn et al., 2011). Two additional studies accrued a larger population of AA patients, but were designed to treat not only AA, but also some other gliomas (Yung et al., 1999; Balmaceda et al., 2008). These 
two studies listed separately the results of treatment of AA as well as the other gliomas and only these trials reported significant efficacy. Recurrent AA remains an incurable brain tumor. The use of TMZ has been recently criticized and there seems to be a return to 20 -year-old nitrosourea based chemotherapy. The chronic toxicity from chemotherapy is often irreversible and may seriously compromise the quality of long-term survival in over $30 \%$ of patients (Sarganas et al., 2012; Grossman et al., 2011). There is an urgent need to investigate new treatment options for recurrent AA.

Antineoplastons (ANPs) A10 and AS2-1 are synthetic derivatives of glutamine, isoglutamine, and phenylacetic acid (Burzynski, 2004). A10 is a synthetic formulation consisting of a 4:1 ratio of phenylacetylglutaminate sodium (PG) and phenylacetylisoglutaminate sodium (isoPG). AS2-1 is a synthetic formulation with a 4:1 ratio of phenylacetate sodium (PN) and PG. An initial phase II study of ANP injections was conducted on 20 patients with astrocytomas in 1988 and it documented objective response in 30\% of patients (S. Burzynski, Kubove, \& B. Burzynski, 1992). A patient diagnosed with diffuse intrinsic pontine (DIPG) AA is currently surviving tumor-free for over 25 years (Burzynski et al., 1992; Hawkins, \& Friedman, 1992). Unfortunately, the study included mixed patient populations; AA and low-grade astrocytoma, newly diagnosed and recurrent tumors, and adults as well as children. Additional phase II clinical trials with ANP were conducted on high-grade glioma in children and adults and the results have been published (S. Burzynski, Janicki, G. Burzynski, \& Marszalek, 2014, pp 2051-2061; S Burzynski, Janicki, G. Burzynski, \& Marszalek, 2014, pp 565-577; S. Burzynski, Janicki, \& G. Burzynski, 2014; S. Burzynski, Janicki, G. Burzynski, Marszalek, \& Brookman, 2014; S. Burzynski, G. Burzynski, \& Janicki, 2014). The report describing the results of phase II study of newly-diagnosed patients with AA was published (S. Burzynski, T. Janicki, G. Burzynski, \& Marszalek, 2015). The treatment was well tolerated and contributed to objective responses and increased survival. With positive preliminary data, we have designed and conducted a single-arm, Phase II study of antineoplaston therapy in 27 adults to assess the efficacy and safety of this treatment in patients who have recurrent and/or progressive AA.

\section{Methods}

\subsection{Patient Population}

The group of 27 patients who were recruited to the study were over 18 years of age with subtotally resected or biopsied tumor prior to therapy, and radiologic evidence of residual or recurrent tumor by gadolinium-enhanced magnetic resonance imaging (MRI) scan performed within 14 days before initiating the study, and no earlier than four weeks following tumor resection. The remaining criteria for admission and removal from the study were the same as for high-grade glioma studies Burzynski et al., 2014, pp 946-956; Burzynski et al., 2014, pp 977-988).

Signed written informed consent was obtained from all subjects before enrollment. Burzynski Research Institute, Inc. (BRI) was the sponsor of the trial and Burzynski Clinic (BC) in Houston, Texas was the single investigator site. The investigational medications were provided free-of-charge.

\subsection{Study Design}

The study was designed as a single-arm, two-stage, interventional phase II trial of ANP (Chang et al., 1999). The study under Protocol BT-15 (FDA IND 43,742) started on March 27, 1996 and closed in November 2007. Subsequently, the protocol was amended by BRI several times; however, none of the amendments altered the aim or design of the original study objectives.

\subsection{Statistical Considerations}

The sample size was calculated based upon the method described by Chang et al. 1999. A response rate to ANP of $\geq 10 \%$ was considered 'of interest,' and the primary endpoint was to determine the overall response rate (confirmed complete response (CR) or partial response (PR)) to ANP. An interim analysis would be conducted after 20 subjects had enrolled in the study. If one or more patients achieved a confirmed radiographic response, an additional 20 subjects would be recruited. Survival was measured from the first day of ANP administration until death from any cause. The distributions of survival and treatment failure were estimated by Kaplan-Meier analysis using MedCalc Statistical Software Version 14.12.0 (MedCalc Software bvba, Ostend, Belgium). All analyses were performed on an intent-to-treat (ITT) population.

\subsection{Administration of the Medications}

ANP was delivered via infusion pump and through subclavian catheter every 4 hours. The details of administration were published recently (Burzynski et al., 2015). The administration of ANP was stopped at the patient's request, worsening of his/her condition, determination of progressive disease or intolerable adverse drug event (ADE). After discontinuation of intravenous (IV) ANP, the patients were advised to consider a maintenance treatment with $0.5 \mathrm{~g}$ capsules of A10 and $0.5 \mathrm{~g}$ capsules of AS2-1 as described before (Burzynski et 
al., 2015).

Medications that were considered necessary for the subjects' welfare and that did not interfere with the evaluation of treatment were given at the discretion of the investigator. The use of corticosteroids was monitored by symptoms and signs and the dose was adjusted to maintain neurological stability. No other anticancer medication was permitted.

After 3 weeks of training at Burzynski Clinic, ANP was administered by the patient/guardian at home under the supervision of their local physician.

\subsection{Evaluation and Follow-Up}

Based on a gadolinium-enhanced MRI, the products of the two greatest perpendicular diameters (LPD) of all lesions were calculated and totaled, providing a baseline evaluation for each study subject. The overall tumor size was also measured including T2 and FLAIR images (Wen et al., 2010; Weller, Cloughesy, Perry, \& Wick, 2013). The baseline provided the reference for determination of the response. Toxicity was evaluated in all patients enrolled in the study (safety population).

In accordance with other Phase II studies conducted at the initiation of this trial, the possible responses to the treatment were CR, PR, SD and PD. CR required the disappearance of all enhancing lesions, sustained for at least 4 weeks, and only physiologic replacement doses of steroids were acceptable. PR required $50 \%$ or higher decrease of the sum of the products of the two largest perpendicular diameters of enhancing lesions and stable or reduced corticosteroid doses. PD was determined when there was over $50 \%$ increase of enhancing lesions or new lesions, and SD was the status between PR and PD. The results of all MRI and PET scans showing objective response were verified by radiologists not affiliated with BRI or BC and their determination of response was accepted. Study subjects were categorized by their overall best response during the course of the treatment. The duration of each response was measured from the date that the criteria for the outcome were first met until the date that PD was first documented. The original protocol required more than 50\% increase of tumor size for PD, but it was amended, and $25 \%$ increase was used and all cases were re-evaluated.

The determination of $1 p 19 q$ deletions and MGMT silencing was not performed at the beginning of the study because they were not available. The additional details of treatment and follow-up were published before (Burzynski et al., 2014, pp 946-956; Burzynski et al., 2014, pp 977-988).

\subsection{Theory}

Patients with recurrent AA have a similar 6-month PFS to patients with recurrent glioblastoma multiforme (GBM) after the change of TMZ regimen. The introduction of targeted therapy helps design new treatment modalities which interrupt signal transmission through RAS and AKT pathways, promote apoptosis and inhibit cell cycle progression at G1/S, S/G2 and G2/M checkpoints (Burzynski \& Patil, 2014). Based on the study of the GBM genome of active ingredients of ANP, PG and PN proposed mechanism of action of ANP is illustrated by Figure 1.

PN interrupts signal transduction through RAS and both PN and PG interrupt transduction through the AKT pathway. PN promotes apoptosis through inhibition of BCL2 and activation of TP53/BAX and VDUPI/Caspase 3. PG blocks expression of AKT2 and increases expression of PTEN. It also decreases expression of ${ }^{\mathrm{BCL}} \mathrm{X}_{\mathrm{L}}$, which is an inhibitor of apoptosis. BAD, which is inhibited by AKT, can now promote apoptosis. In the nucleus PG inhibits MYC and promotes p16 which slows down cell cycle progression at G1/S. PN promotes p21 and slows down cell cycle progression at S/G2 checkpoint. Both PN and PG inhibit checkpoint 1 kinase (CHK1), survivin, and polo-kinase 1 (PLK1), which blocks cell cycle progression through G2/M. The inhibition of cell cycle progression through at G1/S and S/G2 is effected indirectly through cyclin dependent kinases 2/4 (CDK2/4) and cyclin $\mathrm{E} / \mathrm{CDK} 2$. 


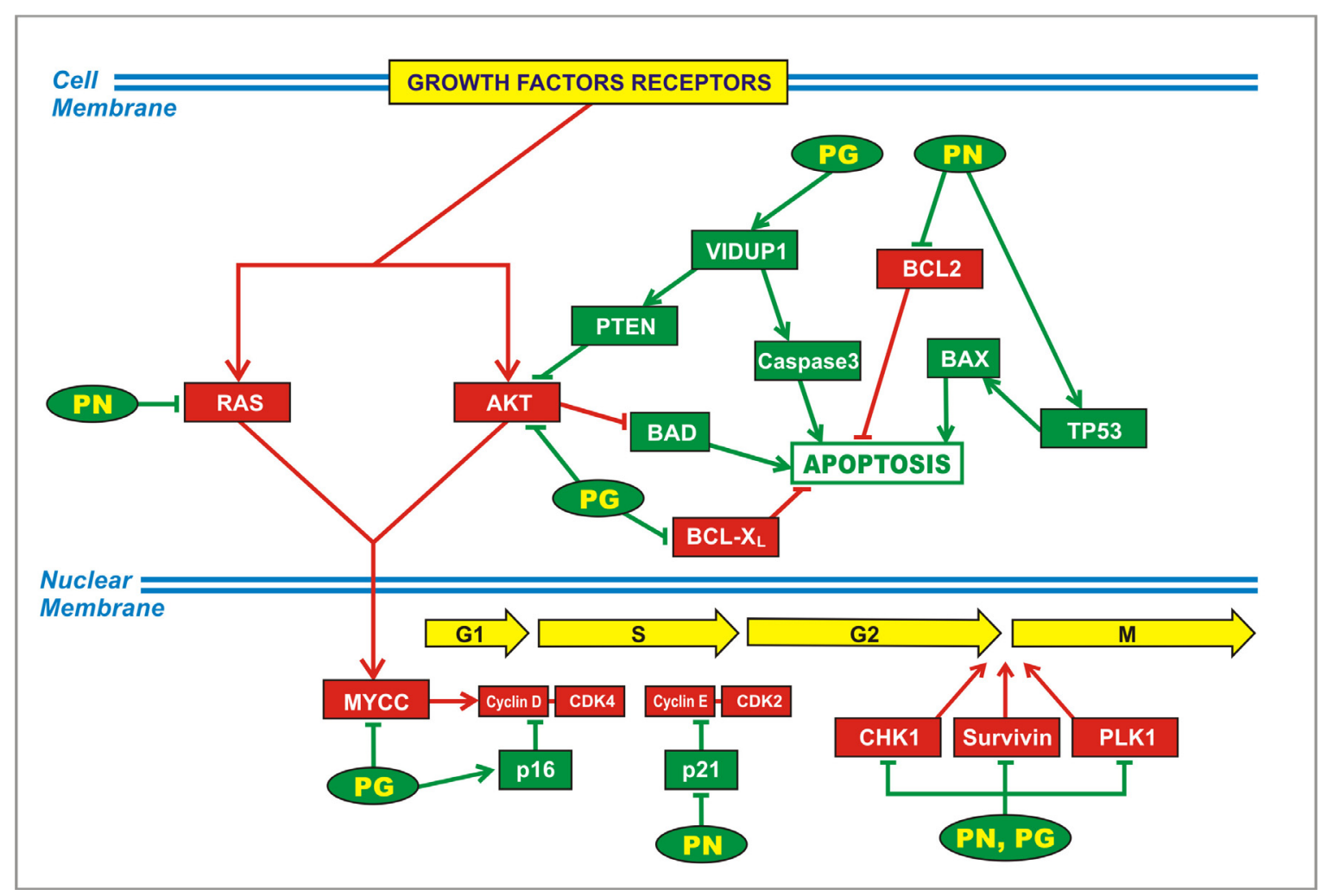

Figure 1. The mechanism of action, which is proposed for active ingredients of Antineoplastons A10 and AS2-1:

PG and PN. PG and PN affect signal transmission through AKT and RAS pathways, promote apoptosis and interrupt cell cycle progression at G1/S and G2/M checkpoints (Burzynski \& Patil, 2014; the abbreviations are explained in the text)

\section{Results}

\subsection{Patient Demographics}

As of December 2008, all subjects were removed from the therapy due to a CR, SD, PD, subject request, worsening clinical condition, or death. The results were analyzed prior to the target enrollment level of $\mathrm{N}=40$, due to sufficient evaluable subjects with a CR and PR.

The 27 candidates (20 men and 7 women) who met enrollment criteria (Table 1) had a median age of 41 years. All subjects were of Caucasian ethnicity. 11 subjects had biopsy only, 4 had partial resection, 12 had subtotal or total resection. 16 subjects had prior chemotherapy. All enrolled subjects received radiation. The overall median tumor size in subjects was $14.58 \mathrm{~cm}^{2}$ (enhancement) (range $0.1-47.67 \mathrm{~cm}^{2}$ ). Patients' characteristics are similar to other studies for recurrent AA. They are compiled and compared with two other studies in Table 2.

Table 1. Study population demographics

\begin{tabular}{llc}
\hline Characteristic & \multicolumn{2}{l}{ Enrolled Subjects $(N=27)$} \\
\hline Age (years) & Number range & $\%$ \\
Median & 41 & \\
Range & $19-57$ & \\
Sex & & 74 \\
Male & 20 & 26 \\
Female & 7 & \\
\hline
\end{tabular}




\begin{tabular}{lll}
\hline Karnofsky performance score at baseline & & \\
60 & 11 & 41 \\
70 & 3 & 11 \\
80 & 7 & 26 \\
90 & 6 & 22 \\
Extent of surgery & & \\
Biopsy only & 11 & 41 \\
Partial resection & 4 & 15 \\
Subtotal or total surgical resection & 12 & 44 \\
Tumor characteristic & & \\
Solitary & 18 & 67 \\
Multifocal & 6 & 22 \\
Multicentric & 3 & 11 \\
Prior chemotherapy & & \\
Yes & 16 & 59 \\
$1^{\text {st }}$ recurrence & 10 & 37 \\
$2^{\text {nd }}$ recurrence & 5 & 18 \\
$3^{\text {rd }}$ recurrence & 1 & 4 \\
Prior radiation & 27 & 100 \\
\hline
\end{tabular}

Table 2. Response to Antineoplaston Treatment Compared to Phase II Studies with chemotherapy for recurrent anaplastic astrocytoma

\begin{tabular}{|c|c|c|c|c|c|c|c|c|}
\hline \multirow[t]{3}{*}{ Author/Treatment } & \multirow{3}{*}{$\begin{array}{l}\text { Patients } \\
\text { Total No. }\end{array}$} & \multicolumn{3}{|c|}{ Efficacy Response (\%) } & \multicolumn{4}{|c|}{ Survival (Probability) } \\
\hline & & $\mathrm{CR}$ & PR & $\mathrm{SD}$ & PFS & & OS & \\
\hline & & & & & $6 \mathrm{mos}$ & $12 \mathrm{mos}$ & $6 \mathrm{mos}$ & $12 \mathrm{mos}$ \\
\hline Chamberlain et al. 2006 Cyclophosphamide & 40 & 0 & 23 & 40 & 30 & 8 & --- & 8 \\
\hline Chamberlain et al. 2008 CPT-11 & 40 & 3 & 10 & 85 & 40 & 5 & --- & 23 \\
\hline Burzynski et al. Antineoplastons A10 and AS2-1 & 27 & 7 & 11 & 22 & 44 & 26 & 63 & 41 \\
\hline
\end{tabular}

Note. $\mathrm{CR}=$ complete response; $\mathrm{OS}=$ overall survival from treatment start; $\mathrm{PFS}=$ progression-free survival; $\mathrm{PR}=$ partial response; $\mathrm{SD}=$ stable disease.

\subsection{Treatment}

In the ITT group the median daily dose of ANP A10 ranged from 2.32 to $8.03 \mathrm{~g} / \mathrm{kg} / \mathrm{d}$ with a median of $6.0 \mathrm{~g} / \mathrm{kg} / \mathrm{d}$. For AS2-1, the median daily dose was $0.25 \mathrm{~g} / \mathrm{kg} / \mathrm{d}$, with a range of 0.16 to $0.41 \mathrm{~g} / \mathrm{kg} / \mathrm{d}$.

The median daily dose of A10 in subjects until OR was observed was $4.81 \mathrm{~g} / \mathrm{kg} / \mathrm{d}$; (range 4.56 to $5.70 \mathrm{~g} / \mathrm{kg} / \mathrm{d}$ ) and the median daily dose of AS2-1 was $0.25 \mathrm{~g} / \mathrm{kg} / \mathrm{d}$; (range 0.21 to $0.32 \mathrm{~g} / \mathrm{kg} / \mathrm{d}$ ). The median time to first OR was 20.9 weeks (range 4.4 to 124.1 weeks).

In the group of subjects with an SD response, the median daily dose of A10 was $6.63 \mathrm{~g} / \mathrm{kg} / \mathrm{d}$ (range 3.90 to 10.97 $\mathrm{g} / \mathrm{kg} / \mathrm{d}$ ) and the median daily dose of AS2-1 was $0.23 \mathrm{~g} / \mathrm{kg} / \mathrm{d}$ (range 0.15 to $0.37 \mathrm{~g} / \mathrm{kg} / \mathrm{d}$ ). The duration of IV ANP therapy in the entire BT-15 trial ranged from 1.1 to 153.4 weeks with a median of 23.1 weeks.

\subsection{Response and Survival}

Out of the 27 enrollees, 2 (7\%) had CR, 3 (11\%) had PR, 6 (22\%) had SD, 10 (37\%) had PD, 6 (22\%) were not further analyzed because they did not complete 12 weeks of therapy nor PD was determined by MRI. 3 out of these 6 patients were removed due to worsening clinical condition, 2 (7\%) were withdrawn from the study per 
subject request and one patient died due to hemorrhage from necrotic tumor, confirmed by autopsy.

The responses in the study compared to two other phase II studies are presented in Table 2. In 7 cases, therapy stopped by subject request, one due to a CR, one due to SD. Eight subjects stopped therapy due to worsening clinical conditions, and 7 subjects halted therapy due to PD. Three subjects died from non-therapy related events during the course of the study. It took a median of 20.8 weeks for OR to be reached (range 4.4-124.1 weeks). The Kaplan-Meier survival curve is shown in Figures 2a and $2 b$.

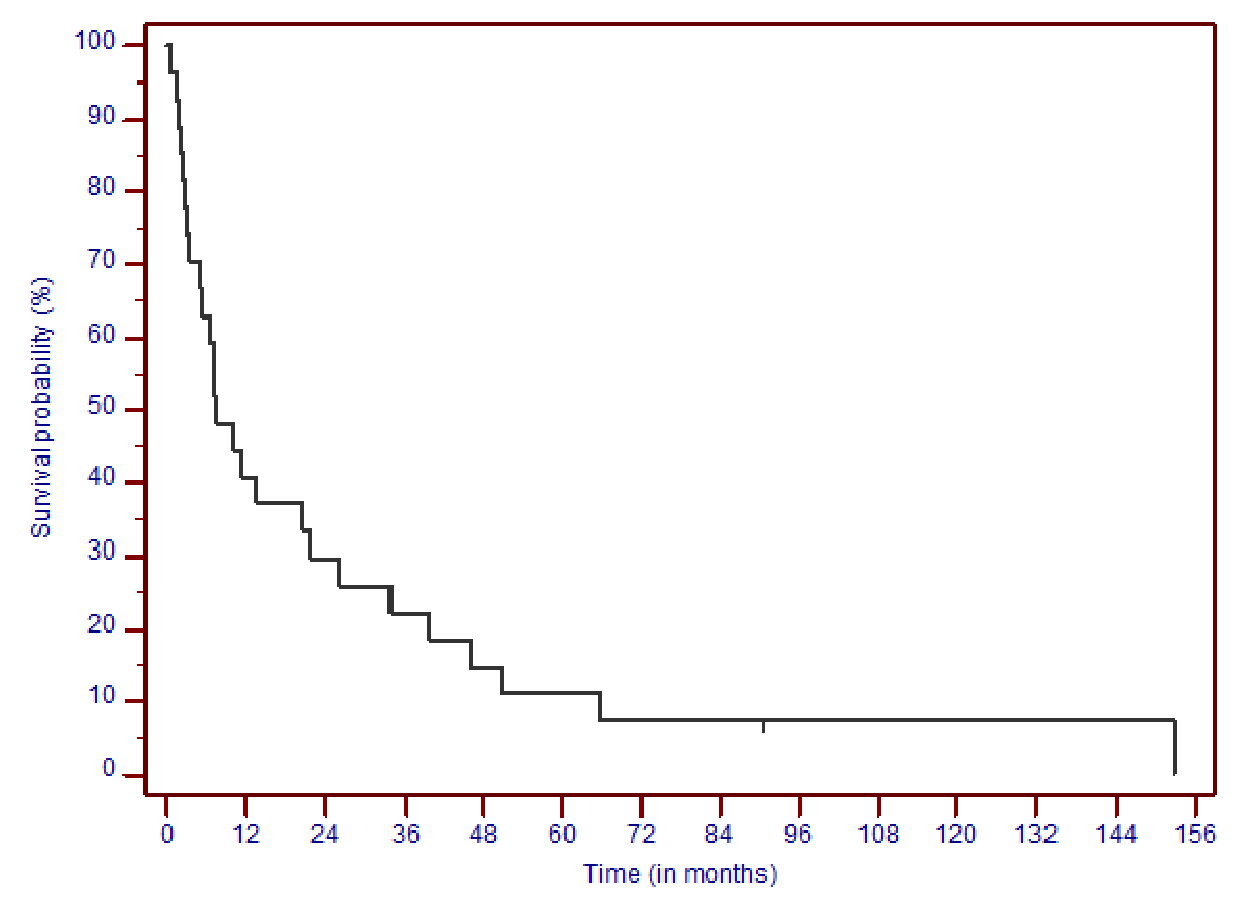

Figure 2a. Kaplan-Meier survival curve - Overall survival from treatment start

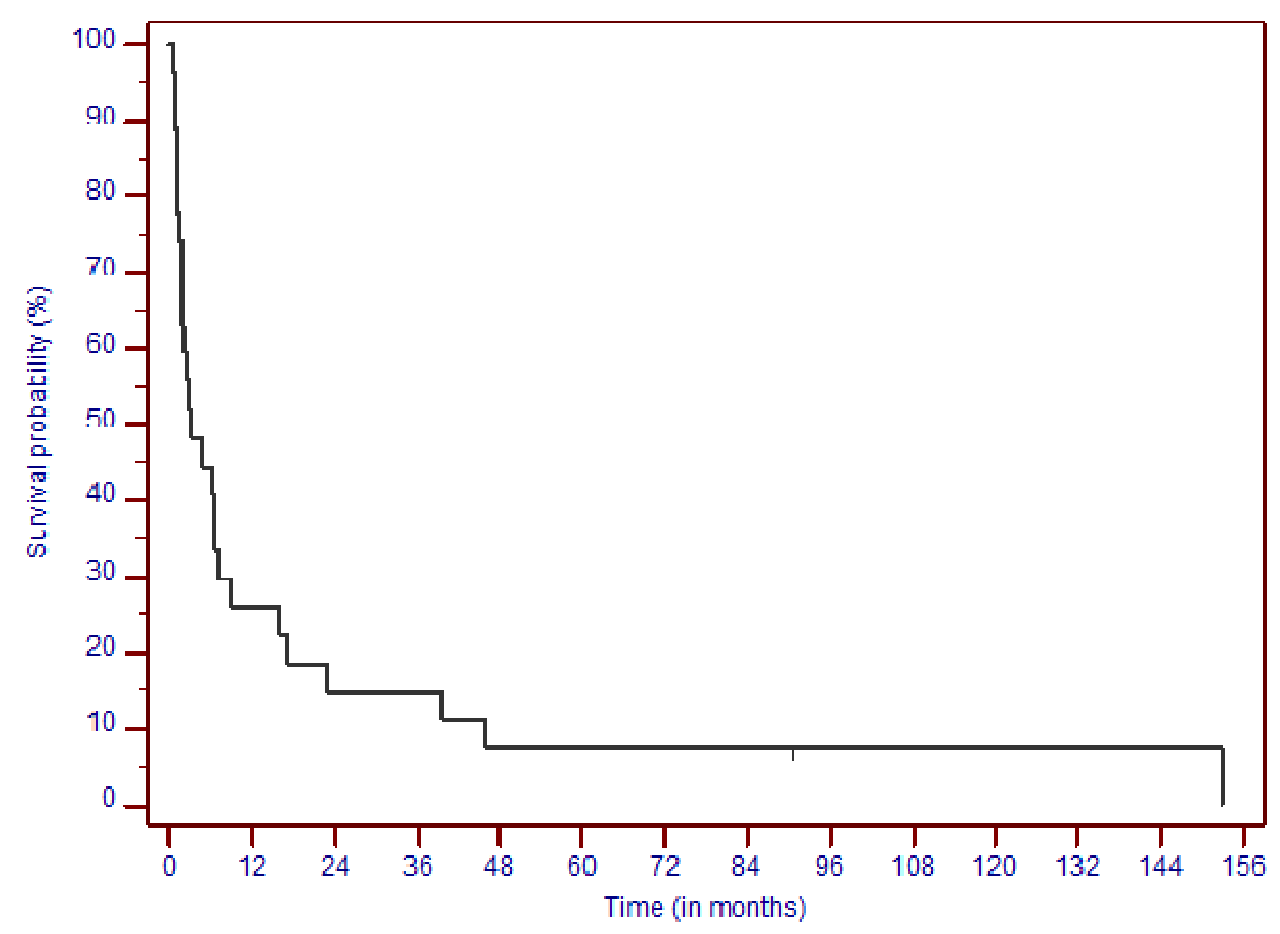

Figure 2b. Kaplan-Meier survival curve - Progression-free survival 
Survival analysis revealed a PFS at 6 months of $44 \%$ and at 12 months $26 \%$. Overall Survival (OS) at 1 year is $41 \%$, at 2 years is $30 \%, 5$ years $11 \%$, and 10 years $7 \%$.

\subsection{Safety and Adverse Drug Events}

Safety assessments were analyzed based upon the total number of enrolled patients in the study $(\mathrm{N}=27)$. Intense monitoring of patient safety was conducted during the first two months of therapy and involved daily direct questioning concerning adverse events, first at the clinic and then followed by daily phone calls during the home administration phase. After two months, telephone contact was conducted on a weekly basis.

A total of 32 ADEs were recorded in 13 subjects: 17 (63\%) Grade 1, 18 (67\%) Grade 2, and 2 (7\%) Grade 3. Grade 4 and 5 ADEs were not reported in the enrolled subjects. A comparison of Grade 3 and 4 ADEs reported by the patients in this study to four other studies is shown in Table 3. There were no long-term ADEs to ANP reported.

Table 3. Incidence of Adverse drug Events (ADEs), Grades 3, 4, or 5, Reported by Patients on antineoplaston treatment compared to other studies of chemotherapy for recurrent anaplastic astrocytoma

\begin{tabular}{|c|c|c|c|c|c|}
\hline \multirow{3}{*}{$\mathrm{ADE}$} & \multirow{2}{*}{$\begin{array}{l}\text { Antineoplastons } \\
N(\%)\end{array}$} & \multicolumn{2}{|c|}{$\begin{array}{l}\text { Chamberlain et al. } 2007 \\
\text { Cyclophosphamide }\end{array}$} & \multicolumn{2}{|c|}{$\begin{array}{l}\text { Chamberlain et al. } 2008 \\
\text { CPT-11 }\end{array}$} \\
\hline & & $N(\%)$ & & $N(\%)$ & \\
\hline & Grade 3 & Grade 3 & Grade 4 & Grade 3 & Grade 5 \\
\hline Hypokalemia & $2(7)$ & & & & \\
\hline Thrombocytopenia & & $5(12)$ & $1(2)$ & $2(5)$ & \\
\hline Neutropenia & & $7(17)$ & $1(2)$ & $1(2)$ & \\
\hline Leukopenia & & $15(37)$ & $2(5)$ & $1(2)$ & \\
\hline Anemia & & $5(12)$ & & & \\
\hline Asthenia & & & & $2(5)$ & \\
\hline Fatigue & & $9(22)$ & & & \\
\hline \multicolumn{6}{|l|}{ Fever } \\
\hline Headache & & $1(2)$ & & & \\
\hline \multicolumn{6}{|l|}{ Peripheral edema } \\
\hline Convulsions & & $1(2)$ & & & \\
\hline \multicolumn{6}{|l|}{ Dizziness } \\
\hline \multicolumn{6}{|l|}{ Somnolence } \\
\hline \multicolumn{6}{|l|}{ Abdominal pain } \\
\hline \multicolumn{6}{|l|}{ Anorexia } \\
\hline Constipation & & $1(2)$ & & & \\
\hline Diarrhea & & & & $3(7)$ & $1(2)$ \\
\hline Nausea & & $3(7)$ & & $2(5)$ & \\
\hline Vomiting & & $2(5)$ & & $4(10)$ & \\
\hline \multicolumn{6}{|l|}{ Pruritus } \\
\hline Infection & & $1(2)$ & & $1(2)$ & \\
\hline Thrombophlebitis & & $1(2)$ & & $1(2)$ & \\
\hline
\end{tabular}

Brain tumor patients frequently receive corticosteroids as part of their therapeutic regimen to reduce peritumoral edema. The infusion of large volume of solutions during ANP treatment, the use of corticosteroids, and the brain tumor itself predisposes the patient to an increase in incident of serum potassium and sodium concentration abnormalities. As the result, three cases of reversible hypokalemia were reported (two were Grade 3, 2.8 and 2.7 
$\mathrm{mEq} / \mathrm{L})$. Three patients had at least one episode of hypernatremia.

In some cases of patients participating in clinical studies with ANP, the blood sample for analysis was taken from the subclavian venous catheter. The results of analysis of these samples were false-positive for hypernatremia, since the catheter was filled with sodium rich ANP.

In this study, there was a single case of hypernatremia of $186 \mathrm{mmol} / \mathrm{L}$ which resulted from withdrawing the sample to be analyzed from the catheter. This event could be classified as a Grade 4 or even a Grade 5 since the patient passed away a half hour later, but an accurate concentration of sodium in serum was never determined. This patient had a total of 38 blood tests with normal serum sodium and only one transient hypernatremia incident of $148 \mathrm{mEq} / \mathrm{L}$ before this incident.

A subclavian venous catheter is required for ANP therapy to permit constant venous access. While the catheters are generally well-tolerated, catheter-related infections can occur when proper handling protocols are not rigorously met. $8(30 \%)$ subjects developed a total of 14 catheter-related infections, all during the period of home-based self-care. Three of the subjects had a Grade 3 infection (11\%) and one had a Grade 4 infection (3\%).

\section{Discussion}

This study and analysis involved patients that had recurrent or progressive AA. Sixteen of the subjects had previously received chemotherapy and five of them failed two and one failed three lines of chemotherapy; thus, the long term toxicity from the prior treatment may have affected their outcome in this trial. The goal was to determine the efficacy and safety of ANP therapy in this patient cohort, despite their prior treatment. The trial was stopped early, due to excess of objective responses $(\mathrm{N}=5)$. Two CRs and three PRs were recorded for $24 \%$ of 21 patients enrolled and evaluated in this study ( $18.5 \%$ for ITT). PFS at 12 months is $26 \%$ and OS $41 \%$.

In comparison to four other phase II studies, this trial shows a similar number of patients. The patients' characteristics are similar except that in both Chamberlain et al. $(2006,2008)$ studies. Prior treatment in the Yung et al. (1999), and Balmaceda et al. (2008) studies consisted of nitrosourea, but in both Chamberlain (2006, 2008) studies, TMZ was used as the initial treatment.

The additional study of Yung et al. (1999), not listed in Table 2, and both studies of Chamberlain et al. (2006, 2008) have a much lower CR rate compared to ANP, but a higher PR rate (Table 3). There were no CRs in the first Chamberlain et al. (2006) study and only 3\% in the second Chamberlain et al. (2008) and 6\% in the Yung et al. (1999) study. Contrary to that, the Balmaceda et al. (2008) trial, not listed in Table 2, shows the highest CR and PR rate. The Yung et al. study and Balmaceda et al. (2008) study can't be compared accurately since the Yung et al. (1999) study accrued patients after their first relapse and the Balmaceda et al. (2008) study excluded patients treated with TMZ.

PFS at 6 months and 12 months is higher in the ANP study compared to the other studies. OS at 12 months is much higher for our study than in the Chamberlain $(2006,2008)$ studies. Table 4 shows a comparison of the incidence of serious ADEs between our study and the two Chamberlain et al. $(2006,2008)$ studies. The comparison reveals a remarkably safer protocol of ANP compared to cyclophosphamide, and CPT11. There were no Grade 4 and 5 ADEs in the ANP study. The percentage of Grade 3 toxicity was very low (7\%) in the ANP study and this toxicity was easily reversible. It is clear that ANP therapy is less disabling than chemotherapy (Table 3).

Of important note is the fact that ANP therapy is safely delivered at home. The low incidence of catheter infections (8 patients, 14 infections) upholds the theory that this treatment is feasible and offers the patient the option of remaining at home while receiving therapy. Such adaptability and flexibility reduced costs related to transportation and hospital stay which are obligatory for radiation and/or chemotherapy.

\section{Conclusion}

It is well known that AAs are associated with a high rate of recurrence after primary therapy and a high mortality rate. The tolerable toxicity and promising objective response rate and survival data of ANP therapy suggest it as an alternative to chemotherapy. Its promise is not just to help patients with AA. Multiple phase II studies of ANP have been completed for malignant gliomas. In addition, a phase III protocol study of ANP therapy with RT versus RT alone in diffuse, intrinsic pontine glioma has been permitted by the FDA. The new studies will also determine the "genomic signature" of the patients. The additional clinical trials can be designed for a combination of ANP and novel targeted agents. Further evaluations and studies will add data specific for each brain tumor and continue to evaluate the long-term toxicity and quality of survival of this therapy. 


\section{Acknowledgments}

The authors express their appreciation to the additional physicians involved in the care of the patients: Drs. Robert A. Weaver, Robert I. Lewy, Eva Kubove, and Barbara Szymkowski. Preparation of the manuscript was provided by Carolyn Powers and Jennifer Pineda.

\section{Disclosure Statement}

The authors have no conflicts of interest to declare.

\section{References}

Balmaceda, C., Peereboom, D., Pannullo, S., Cheung, Y. K., Fisher, P. G., Alavi, J., ... Fine, R. L. (2008). Multi-institutional phase II study of temozolomide administered twice daily in the treatment of recurrent high-grade gliomas. Cancer, 112(5), 1139-1146. http://dx.doi.org/10.1002/cncr.23167

Bogdahn, U., Hau, P., Stockhammer, G., Venkataramana, N. K., Mahapatra, A. K., Suri, A., ... Schlingensiepen, K. H. (2011). Targeted therapy for high-grade glioma with the TGF- $\beta 2$ inhibitor trabedersen: results of a randomized and controlled phase IIb study. Neuro-oncology, 13(1), 132-142. http://dx.doi.org/10.1093/neuonc/noq142

Burzynski, S. R. (2004). The present state of antineoplaston research (1). Integrative Cancer Therapies, 3(1), 47-58.

Burzynski, S. R., Burzynski, G. S., \& Janicki, T. J. (2014). Recurrent glioblastoma multiforme - A strategy for long-term survival. Journal of Cancer Therapy, 5(10), 957-976. http://dx.doi.org/10.4236/jct.2014.510101

Burzynski, S. R., Janicki, T. J., \& Burzynski, G. S. (2014). A phase II study of Antineoplastons A10 and AS2-1 in adult patients with recurrent glioblastoma multiforme: Final report (Protocol BT-21). Journal of Cancer Therapy, 5(10), 946-956. http://dx.doi.org/10.4236/jct.2014.510100

Burzynski, S. R., Janicki, T. J., Burzynski, G. S., \& Marszalek, A. (2014). The response and survival of children with recurrent diffuse intrinsic pontine glioma based on phase II study of Antineoplastons A10 and AS2-1 in patients with brainstem glioma. Child's Nervous System, 30(12), 2051-2061. http://dx.doi.org/10.1007/s00381-014-2401-z

Burzynski, S. R., Janicki, T. J., Burzynski, G. S., \& Marszalek, A. (2014). A phase II study of Antineoplastons A10 and AS2-1 in children with high-grade glioma. Final report (Protocol BT-06) and review of recent trials. Journal of Cancer Therapy, 5(6), 565-577. http://dx.doi.org/10.4236/jct.2014.56065

Burzynski, S. R., Janicki, T. J., Burzynski, G. S., \& Marszalek, A. (2015). A phase II study of Antineoplastons A10 and AS2-1 in adult patients with newly-diagnosed anaplastic astrocytoma. Final report (Protocol BT-08). Cancer \& Clinical Oncology, 4(1), 28-38. http://dx.doi.org/10.5539/cco.v4n1p28

Burzynski, S. R., Janicki, T. J., Burzynski, G. S., Marszalek, A., \& Brookman, S. (2014). A phase II study of Antineoplastons A10 and AS2-1 in children with recurrent, refractory or progressive primary brain tumors Final report (Protocol BT-22). Journal of Cancer Therapy, 5(10), 977-988. http://dx.doi.org/10.4236/jct.2014.510102

Burzynski, S. R., Kubove, E., \& Burzynski, B. (1992). Phase II clinical trials of antineoplastons A10 and AS2-1 infusions in astrocytoma. In D. Adam (Ed.), Recent advances in chemotherapy. Munich, Germany: Futuramed Publishers.

Burzynski, S. R., \& Patil, S. (2014). The effect of antineoplastons A10 and AS2-1 and metabolites of sodium phenylbutyrate on gene expression in glioblastoma multiforme. Journal of Cancer Therapy, 5(10), 929-945. http://dx.doi.org/10.4236/jct.2014.510099

Chamberlain, M. C., Tsao-Wei, D. D., Blumenthal, D. T., \& Glantz, M. J. (2008). Salvage chemotherapy with CPT-11 for recurrent temozolomide-refractory anaplastic astrocytoma. Cancer, 112(9), 2038-2045.

Chamberlain, M. C., Tsao-Wei, D. D., \& Groshen, S. (2006). Salvage chemotherapy with cyclophosphamide for recurrent temozolomide-refractory anaplastic astrocytoma. Cancer, 106(1), 172-179. http://dx.doi.org/10.1002/cncr.21582

Chang, S. M., Kuhn, J. G., Robins, H. I., Schold, S. C., Spence, A. M., Berger, M. S., ... Prados, M. D. (1999). Phase II study of phenylacetate in patients with recurrent malignant glioma: a North American Brain Tumor Consortium report. Journal of Clinical Oncology, 17(3), 984-990.

de Groot, J. F., Lamborn, K. R., Chang, S. M., Gilbert, M. R., Cloughesy, T. F., Aldape, K., .. Wen, P. Y. (2011). 
Phase II study of aflibercept in recurrent malignant glioma: A North American Brain Tumor Consortium study. Journal of Clinical Oncology, 29(19), 2689-2695. http://dx.doi.org/10.1200/JCO.2010.34.1636

Dinnes, J., Cave, C., Huang, S., \& Milne, R. (2002). A rapid and systematic review of the effectiveness of temozolomide for the treatment of recurrent malignant glioma. British Journal of Cancer, 86(4), 501-505. http://dx.doi.org/10.1038/sj.bjc.6600135

Franceschi, E., Cavallo, G., Lonardi, S., Magrini, E., Tosoni, A., Grosso, D., \& Brandes, A. A. (2007). Gefitinib in patients with progressive high-grade gliomas: a multicentre phase II study by Gruppo Italiano Cooperativo di Neuro-Oncologia (GICNO). British Journal of Cancer, 96(7), 1047-1051. http://dx.doi.org/10.1038/sj.bjc.6603669

Grossman, S. A., Ye, X., Lesser, G., Sloan, A., Carraway, H., Desideri, S., \& Piantadosi, S. (2011). Immunosuppression in patients with high-grade gliomas treated with radiation and temozolomide. Clinical Cancer Research, 17(16), 5473-5480. http://dx.doi.org/10.1158/1078-0432.CCR-11-0774

Hawkins, M. J., \& Friedman, M. A. (1992). National Cancer Institute's evaluation of unconventional cancer treatments. Journal of the National Cancer Institute, 84(22), 1699-1702. http://dx.doi.org/10.1093/jnci/84.22.1699

Kirby, S., Gertler, S. Z., Mason, W., Watling, C., Forsyth, P., Aniagolu, J., ... Eisenhauer, E. A. (2005). Phase 2 study of T138067-sodium in patients with malignant glioma: Trial of the National Cancer Institute of $\begin{array}{lllll}\text { Canada Clinical Trials } & \text { Group. } & \text { Neuro-oncology, } & 7(2), & 183-188 .\end{array}$ http://dx.doi.org/10.1215/S1152851704000602

Louis, D. N., Ohgaki, H., Wiestler, O. D., Cavenee, W. K., Burger, P. C., Jouvet, A., ... Kleihues, P. (2007). The 2007 WHO classification of tumours of the central nervous system. Acta Neuropathologica, 114(2), 97-109. http://dx.doi.org/10.1007/s00401-007-0243-4

Mehta, M. P., Vogelbaum, M., Chang, S., \& Patel, N. (2011). Neoplasms of the central nervous system. In V.T. DeVita, T.S. Lawrence, \& S.A. Rosenberg, (Eds.), Cancer: Principles and practice of oncology $\left(9^{\text {th }} \mathrm{ed}\right.$., 1700-1749). Philadelphia, PA: Lippincott Williams \& Wilkins.

Neyns, B., Chaskis, C., Joosens, E., Menten, J., D'Hondt, L., Branle, F., ... Michotte, A. (2008). A multicenter cohort study of dose-dense temozolomide (21 of 28 days) for the treatment of recurrent anaplastic

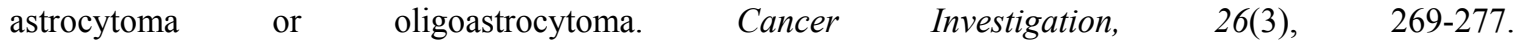
http://dx.doi.org/10.1080/07357900701708393

Nishikawa, R., Shibui, S., Maruno, M., Sugiyama, K., Sato, S., Fujimaki, T., ... Matsutani, M. (2006). Efficacy and safety of monotherapy with temozolomide in patients with anaplastic astrocytoma at first relapse--a phase II clinical study. Gan To Kagaku Ryoho. Cancer \& Chemotherapy, 33(9), 1279-1285.

Ostrom, Q. T., Gittleman, H., Liao, P., Rouse, C., Chen, Y., Dowling, J., ... Barnholtz-Sloan, J. (2014). CBTRUS statistical report: Primary brain and central nervous system tumors diagnosed in the United States in 2007-2011. Neuro-oncology, 16(Suppl 4), iv1-iv63. http://dx.doi.org/10.1093/neuonc/nou223

Robins, H. I., Chang, S. M., Prados, M. D., Yung, W. K., Hess, K., Schiff, D., ... Mehta, M. (2002). A phase II trial of thymidine and carboplatin for recurrent malignant glioma: a North American Brain Tumor Consortium Study. Neuro-oncology, 4(2), 109-114. http://dx.doi.org/10.1215/15228517-4-2-109

Sarganas, G., Orzechowski, H. D., Klimpel, A., Thomae, M., Kauffmann, W., Herbst, H., ... Garbe, E. (2012). Severe sustained cholestatic hepatitis following temozolomide in a patient with glioblastoma multiforme: case study and review of data from the FDA adverse event reporting system. Neuro-oncology, 14(5), 541-546. http://dx.doi.org/10.1093/neuonc/nos056

SAS Institute: 1989-2010 JMP, version 8.0.2. SAS Institute, Cary, NC.

Spence, A. M., Peterson, R. A., Scharnhorst, J. D., Silbergeld, D. L., \& Rostomily, R. C. (2004). Phase II study of concurrent continuous Temozolomide (TMZ) and Tamoxifen (TMX) for recurrent malignant astrocytic

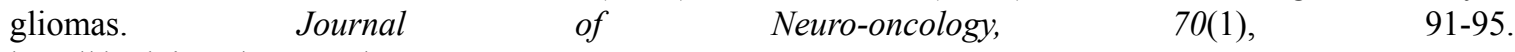
http://dx.doi.org/10.1023/B:NEON.0000040837.68411.97

Weller, M., Cloughesy, T., Perry, J. R., \& Wick, W. (2013). Standards of care for treatment of recurrent glioblastoma - are we there yet? Neuro-oncology, 15(1), 4-27. http://dx.doi.org/10.1093/neuonc/nos273

Wen, P. Y., Macdonald, D. R., Reardon, D. A., Cloughesy, T. F., Sorensen, A. G., Galanis, E., ... Chang, S. M. (2010). Updated response assessment criteria for high-grade gliomas: Response assessment in 
neuro-oncology working group. Journal of Clinical Oncology, 28(11), 1963-1972. http://dx.doi.org/10.1200/JCO.2009.26.3541

Yung, W. K., Prados, M. D., Yaya-Tur, R., Rosenfeld, S. S., Brada, M., Friedman, H. S., ... Levin, V. A. (1999). Multicenter phase II trial of temozolomide in patients with anaplastic astrocytoma or anaplastic oligoastrocytoma at first relapse. Temodal Brain Tumor Group. Journal of Clinical Oncology, 17(9), 2762-2771.

\section{Copyrights}

Copyright for this article is retained by the author(s), with first publication rights granted to the journal.

This is an open-access article distributed under the terms and conditions of the Creative Commons Attribution license (http://creativecommons.org/licenses/by/3.0/). 